\title{
Résumé de la tuberculose au Canada en 2013
}

\author{
Gallant $\mathbf{V}^{1}$, McGuire $\mathbf{M}^{1}$, Ogunnaike-Cooke $\mathrm{S}^{1 *}$ \\ ${ }^{1}$ Section de la surveillance du VIH/sida et de la tuberculose, Agence de la santé publique du Canada, Ottawa (Ontario) \\ * Correspondance : Susanna.Ogunnaike-Cooke@phac-aspc.gc.ca
}

\section{Résumé}

Contexte : L'Agence de la santé publique du Canada (l'Agence) surveille la tuberculose active par l'entremise du Système canadien de déclaration des cas de tuberculose (SCDCT). Elle utilise les données et les rapports de surveillance de la tuberculose pour observer les progrès réalisés envers l'atteinte de la cible que s'est fixée le Canada, soit de prévenir et de contrôler la transmission de la tuberculose, tel qu'il est décrit dans le document intitulé Prévention et contrôle de la tuberculose au Canada - Un cadre d'action fédéral.

Objectif : Fournir un aperçu du nombre préliminaire de cas signalés de tuberculose active (nouveaux cas et cas de retraitement) ainsi que des taux d'incidence au Canada en 2013.

Méthodologie : Chaque année, les autorités de la santé publique provinciales et territoriales soumettent volontairement des données au SCDCT sur tous les cas de tuberculose qui répondent à la définition de cas dans le cadre de la surveillance nationale. Ces données sont analysées par l'Agence. Les résultats de l'analyse sont validés par la province ou le territoire concerné et sont publiés annuellement dans la série intitulée La tuberculose au Canada - Prédiffusion.

Résultats : Au Canada, 1640 nouveaux cas de tuberculose active et cas de retraitement de la tuberculose ont été déclarés en 2013, ce qui correspond à un taux d'incidence global de 4,7 cas pour 100000 habitants. La répartition générale des cas selon le sexe et le groupe d'âge était semblable à celle des années précédentes. Même si les personnes âgées de 25 à 34 ans représentaient le pourcentage le plus élevé de cas déclarés (16\%), le taux d'incidence le plus élevé a été observé pour les personnes âgées de 75 ans ou plus. Les taux en Colombie-Britannique, au Manitoba, en Saskatchewan, au Nunavut et dans les Territoires du Nord-Ouest étaient supérieurs au taux canadien, et c'est au Nunavut que le taux d'incidence était le plus élevé. Les personnes nées à l'étranger ont continué à représenter la majorité des cas de tuberculose signalés, mais le taux d'incidence pour 100000 habitants est resté le plus élevé chez les Autochtones nés au Canada.

Conclusion : Les données préliminaires de 2013 indiquent qu'il n'y a pas eu de changements notables dans le nombre de cas de tuberculose signalés ou dans le taux d'incidence global au Canada en comparaison des années précédentes. De même, aucun changement n'a été relevé quant à la répartition des cas par province ou territoire, par groupe d'âge ou par sexe.

\section{Introduction}

L'Agence de la santé publique du Canada (l'Agence) assure le leadership à l'échelle nationale pour ce qui est des aspects de la santé publique concernant la tuberculose et travaille en collaboration avec les partenaires nationaux et internationaux pour s'attaquer à la prévention et à l'endiguement de la tuberculose. Deux des principales responsabilités de l'Agence sont la surveillance et le rapportage sur la tuberculose par l'entremise d'un système national de surveillance, à savoir le Système canadien de déclaration des cas de tuberculose (SCDCT), qui est le fruit de la collaboration entre les ministères de la Santé des provinces et des territoires. 
Chaque année, l'Agence publie un résumé intitulé La tuberculose au Canada - Prédiffusion (1), qui permet un accès rapide aux données nationales de surveillance de la tuberculose. Tous les trois ans, un rapport de surveillance exhaustif est publié sur La tuberculose au Canada (2). L'Agence utilise ces données et les rapports de surveillance de la tuberculose pour observer les progrès réalisés envers l'atteinte de la cible que s'est fixée le Canada, soit de prévenir et de contrôler la transmission de la tuberculose, tel qu'il est décrit dans le document intitulé Prévention et contrôle de la tuberculose au Canada - Un cadre d'action fédéral (3).

Le présent article fournit un aperçu du nombre préliminaire de cas signalés de tuberculose active (nouveaux cas et cas de retraitement) ainsi que des taux d'incidence au Canada en 2013 (2).

\section{Contexte}

Comme la déclaration des cas de tuberculose est obligatoire dans l'ensemble des provinces et des territoires, les professionnels de la santé doivent les déclarer en vertu de la loi à leur ministère de la Santé. La tuberculose est une maladie à déclaration obligatoire depuis 1924, ce qui signifie qu'elle est surveillée de façon prioritaire à l'échelle nationale. Or, aucune loi n'oblige les provinces ou les territoires à déclarer les cas et les résultats aux fins de surveillance nationale. Toutefois, dans un esprit de collaboration visant à comprendre le fardeau de la tuberculose au pays, les autorités de la santé publique provinciales et territoriales soumettent volontairement des données sur les cas de tuberculose qui répondent à la définition de cas aux fins de surveillance nationale (consulter l'encadré ci-dessous) (4).

\section{Définitions nationales des cas de tuberculose et des cas de retraitement}

\section{Tuberculose}

\section{Cas confirmé}

- Cas confirmé en laboratoire : Cas d'infection par le complexe Mycobacterium tuberculosis mis en évidence par culture, en particulier M. tuberculosis, M. africanum, M. canetti, M. caprae, M. microti, M. pinnipedii ou M. bovis (à l'exception de la souche du bacille de Calmette-Guérin [BCG] de M. bovis).

- Cas confirmé cliniquement : Si l'on ne possède pas de preuves bactériologiques, cas présentant des signes cliniques compatibles avec une tuberculose active, par exemple cas où :

I. les modifications de la radiographie des poumons sont compatibles avec le diagnostic de tuberculose active;

II. un diagnostic de tuberculose non respiratoire active (méninges, os, rein, ganglions lymphatiques périphériques, etc.) a été posé;

III. un examen anatomopathologique ou une autopsie confirme le diagnostic de tuberculose active;

IV. une réponse favorable à un essai thérapeutique d'antituberculeux a été obtenue.

\section{Nouveaux cas et cas de retraitement de la tuberculose}

\section{- $\quad$ Nouveau cas}

Aucune preuve ou absence d'antécédents précis de tuberculose active.

\section{- Cas de retraitement}

I. Preuve accompagnée de pièces justificatives et antécédentes adéquats de tuberculose active qui a été déclarée guérie ou dont le traitement est considéré comme terminé selon les normes actuelles;

II. Laps d'au moins 6 mois depuis le dernier jour du traitement précédent;

III. Diagnostic d'un épisode subséquent de tuberculose qui répond à la définition de cas de tuberculose active.

OU

I. Preuve accompagnée de pièces justificatives et antécédentes adéquats de tuberculose active qui 
ne peut être déclarée guérie ou dont le traitement ne peut être considéré comme terminé selon les normes actuelles;

II. Tuberculose inactive pendant 6 mois ou plus après le dernier jour du traitement précédent;

III. Diagnostic d'un épisode subséquent de tuberculose qui répond à la définition de cas de tuberculose active.

OU

I. Preuve accompagnée de pièces justificatives et antécédentes adéquats de tuberculose active qui ne peut être déclarée guérie ou dont le traitement ne peut être considéré comme terminé selon les normes actuelles;

II. Tuberculose inactive pendant 6 mois ou plus après le dernier jour du traitement précédent;

III. Diagnostic d'un épisode subséquent de tuberculose qui répond à la définition de cas de tuberculose active.

\section{Méthodologie}

Administré par l'Agence, le (SCDT) est un système de surveillance national fondé sur les cas, qui conserve certaines données non nominatives sur les personnes ayant reçu un diagnostic de tuberculose active. Ces données comprennent entre autres de l'information sur les caractéristiques démographiques ( $p$. ex. âge, sexe, origine, statut d'immigration), l'état clinique, le diagnostic et le traitement. Les renseignements sur les facteurs de risque (p. ex. sérologie $\mathrm{VIH}$ ) et les résultats des traitements ont été recueillis, mais ne font pas partie du présent article. La collecte des données s'effectue au moyen d'un formulaire de déclaration standard rempli manuellement ou transmis électroniquement.

Le " taux d'incidence » de la tuberculose s'entend ici du nombre de personnes ayant nouvellement reçu un diagnostic de tuberculose active (nouveaux cas et cas de retraitement) pour chaque année de déclaration et pour 100000 habitants. Les taux représentent le nombre de cas déclarés par année civile pour 100000 habitants. Aucune procédure statistique n'a été utilisée pour les analyses comparatives, et aucune technique statistique n'a été appliquée pour tenir compte des données manquantes. Les taux d'incidence chez les personnes nées à l'étranger sont fondés sur les estimations de la population tirées du recensement de la population canadienne. La population non autochtone née au Canada représente l'ensemble de la population canadienne moins les personnes nées à l'étranger et les Autochtones. La classification des diagnostics de tuberculose au Canada repose sur les $9^{\mathrm{e}}$ et $10^{\mathrm{e}}$ éditions de la Classification internationale des maladies $(5,6)$.

\section{Résultats}

Au Canada, 1640 nouveaux cas de tuberculose active et cas de retraitement de la tuberculose ont été déclarés en 2013, soit un taux d'incidence global de 4,7 cas pour 100000 habitants, ce qui est légèrement plus faible que le nombre de cas déclarés en $2012(n=1699)$ et que le taux d'incidence en $2012(4,9$ cas pour 100000 habitants). Le taux annuel d'incidence de la tuberculose est inférieur à 5 cas pour 100000 habitats depuis 2007 (Figure 1). 
Figure 1: Nombre de cas de tuberculose déclarés et taux d'incidence pour 100000 habitants Canada : 2003 à 2013

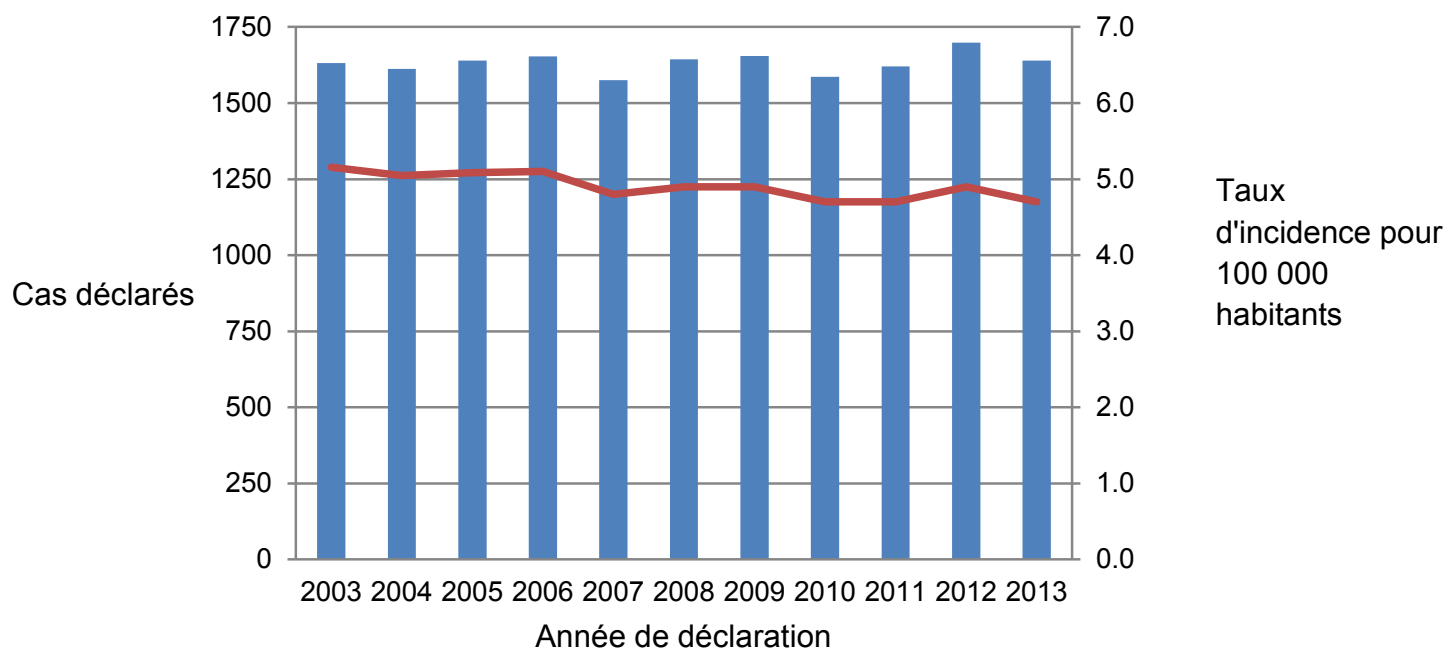

La tuberculose active peut être classée comme maladie respiratoire ou maladie non respiratoire. La tuberculose respiratoire comprend la tuberculose pulmonaire, la tuberculose pleurale, la tuberculose des ganglions lymphatiques endothoraciques et des ganglions lymphatiques du médiastin, ainsi que la tuberculose du larynx, du nasopharynx, du nez ou des sinus. La tuberculose non respiratoire désigne tous les autres sièges de la maladie (3). En 2013, à l'image des années précédentes, $77 \%$ des cas ont reçu un diagnostic de tuberculose respiratoire et $23 \%$, un diagnostic de tuberculose non respiratoire (miliaire, système nerveux central, ganglions lymphatiques périphériques ou autre).

\section{Distribution par âge et par sexe}

En 2013, comme au cours des années précédentes, les hommes représentaient la majorité des cas déclarés, soit $55 \%$, pour un taux d'incidence global de 5,2 cas pour 100000 hommes. À titre de comparaison, les femmes représentaient $45 \%$ de tous les cas signalés, pour un taux d'incidence de 4,2 cas pour 100000 femmes.

En 2013, les personnes âgées de 25 à 34 ans représentaient le plus fort pourcentage des cas déclarés (16 \%). Par ailleurs, $7 \%$ des cas déclarés étaient des enfants de moins de 15 ans. Le taux d'incidence le plus élevé a été observé dans la population âgée de 75 ans et plus, soit 9,3 cas pour 100000 habitants (Figure 2). 
Figure 2 : Taux d’incidence de la tuberculose au Canada en 2013 pour 100000 habitants, par groupe d'âge

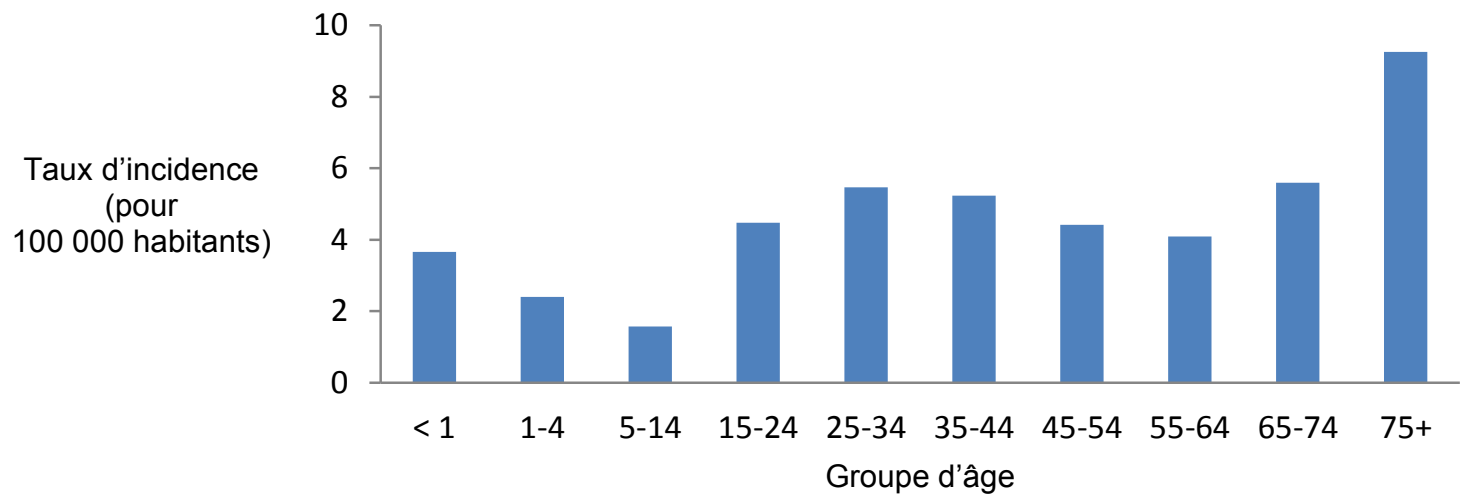

\section{Distribution géographique}

En 2013, aucun cas de tuberculose n'a été déclaré à l'Île-du-Prince-Édouard. Les taux d'incidence dans les autres provinces atlantiques, de même qu'en Alberta, en Ontario et au Québec, étaient tous équivalents ou inférieurs au taux canadien de 4,7 cas pour 100000 habitants (Figure 3). Les taux d'incidence en ColombieBritannique, au Manitoba, en Saskatchewan, au Nunavut, dans les Territoires du Nord-Ouest et au Yukon étaient plus élevés que le taux canadien. Le Nunavut présentait encore une fois le taux d'incidence le plus élevé, soit 143,3 cas pour 100000 habitants. Depuis 2004, le taux d'incidence au Nunavut est le plus élevé Canada. II a atteint un sommet en 2010, à 299,8 cas pour 100000 habitants. Par contre, le taux d'incidence au Nunavut était plus faible en 2013 qu'en 2012 (230,5 cas pour 100000 habitants). Cette baisse correspond à 29 cas en moins en 2013 par rapport à 2012.

Figure 3 : Taux d'incidence de la tuberculose pour 100000 habitants par province ou territoire en 2013

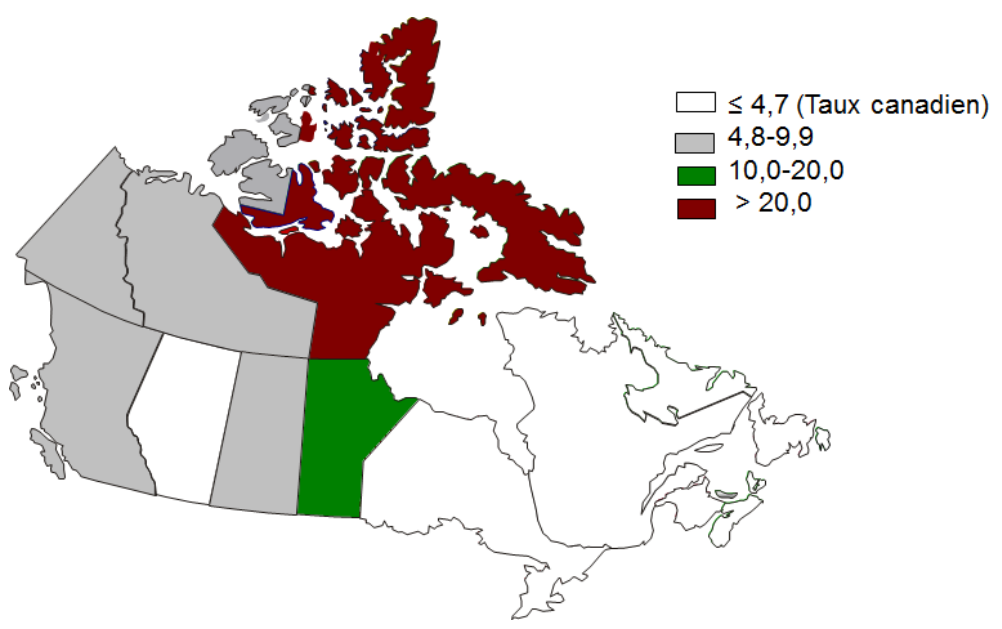

\section{Distribution selon l'origine}

Les personnes nées à l'étranger et les Autochtones nés au Canada étaient toujours représentés de façon disproportionnée parmi les cas déclarés de tuberculose en 2013. Des données sur l'origine étaient accessibles pour 1611 (98\%) des 1640 cas déclarés en 2013. Les personnes nées à l'étranger, qui composaient environ $22 \%$ de la population canadienne totale en 2013 , représentaient $71 \%$ des cas déclarés d'origine connue 
( $n=1146$ ), avec un taux d'incidence correspondant de 14,8 cas pour 100000 habitants. Les Autochtones nés au Canada, qui composaient $4 \%$ de la population canadienne totale en 2013 , représentaient $19 \%$ des cas déclarés d'origine connue ( $n=309$ ), avec un taux d'incidence correspondant de 19,9 cas pour 100000 habitants. Les nonAutochtones nés au Canada représentaient le plus faible pourcentage de cas déclarés, soit $10 \%(n=155)$, avec un taux d'incidence correspondant de 0,6 cas pour 100000 habitants (Figure 4).

Figure 4 : Pourcentage des cas de tuberculose déclarés au Canada selon l’origine, de 2003 à 2013

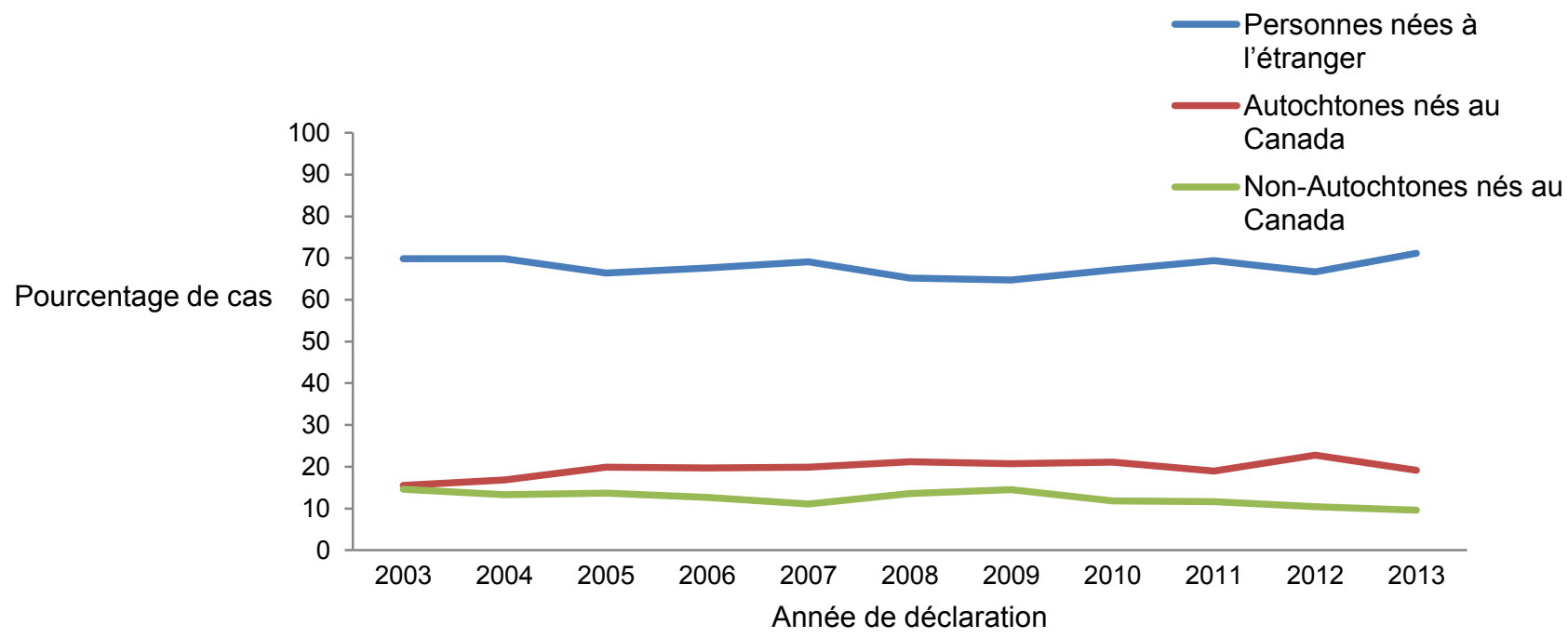

Les Autochtones nés au Canada sont répartis dans trois populations distinctes : les Premières nations, les Inuits et les Métis. En 2013, parmi les 309 cas déclarés chez les Autochtones nés au Canada, 200 (65\%) étaient des membres des Premières nations, 91 (29\%) étaient des Inuits et 18 (6\%) étaient des Métis. Le taux d'incidence parmi les Métis était de 3,3 cas pour 100000 habitants, ce qui est inférieur au taux d'incidence global pour le Canada, qui est de 4,7 cas pour 100000 habitants. En comparaison, le taux d'incidence chez les membres des Premières nations, qui s'élevait à 21,8 cas pour 100000 habitants, était près de cinq fois supérieures au taux global canadien. Le taux d'incidence le plus élevé a toutefois été observé chez les Inuits, soit 154,2 cas pour 100000 habitants.

\section{Discussion}

Dans l'ensemble, le Canada présente l'un des taux de tuberculose les plus faibles au monde. Par rapport à 2012, les données préliminaires de 2013 indiquent qu'il n'y a pas eu de changements notables quant au nombre de cas de tuberculose signalés ou au taux d'incidence global au Canada. De même, aucun changement n'a été relevé quant à la répartition des cas par province ou territoire, par groupe d'âge ou par sexe. Les personnes nées à l'étranger ont continué à représenter la majorité des cas de tuberculose signalés, mais le taux d'incidence pour 100000 habitants est resté le plus élevé chez les Autochtones nés au Canada.

Plusieurs facteurs doivent être pris en considération dans le cadre de l'interprétation de ces données. Le Système canadien de déclaration des cas de tuberculose est un système de surveillance passif qui repose sur les données recueillies de façon rétrospective à partir des dossiers médicaux et des résultats de laboratoire. Par conséquent, il est difficile d'établir clairement si toutes les personnes atteintes de tuberculose ont été répertoriées. Toutefois, l'Organisation mondiale de la Santé (OMS) estime que le système de surveillance du Canada a un taux de détection de $92 \%$ et que l'étendue de l'incertitude est de $85 \%$ à $100 \%$ (7). 
Les données de surveillance figurant dans ce rapport peuvent présenter des erreurs occasionnelles de codage, de déclaration ou de traitement. En général, les données sur les caractéristiques démographiques, l'état clinique et les résultats de laboratoire sont complètes pour plus de $95 \%$ des cas déclarés. Dans certains systèmes de déclaration provinciaux et territoriaux, les cas de tuberculose chez les membres des Premières nations n'ont pu être déclarés séparément des cas chez les Métis. Par conséquent, les taux d'incidence chez les Métis doivent être interprétés avec prudence.

Enfin, les résultats de cette analyse sont provisoires. La mise à jour du nombre de cas de tuberculose et des taux d'incidence pour 2013 sera présentée dans la prochaine génération de rapport de surveillance La tuberculose au Canada.

En conclusion, une compréhension approfondie des tendances épidémiologiques de la tuberculose au Canada fait partie intégrante du suivi des progrès réalisés en ce qui concerne l'objectif national de réduction du fardeau de la tuberculose. À mesure qu'évoluera l'épidémiologie de la tuberculose à l'échelle du Canada et à l'échelle mondiale, l'Agence continuera de travailler avec ses partenaires dans le but d'améliorer la qualité et la nature des données ainsi qu'à en faire état rapidement.

\section{Remerciements}

La Division de surveillance de la santé et de l'épidémiologie de l'Agence de la santé publique du Canada tient à remercier les programmes provinciaux et territoriaux de lutte antituberculeuse et leurs équipes de leur contribution et de leur participation au Système canadien de déclaration des cas de tuberculose.

\section{Conflit d'intérêts}

\section{Aucun}

\section{Financement}

Le présent rapport a été financé par l'Agence de la santé publique du Canada.

\section{Références}

(1) Public Health Agency of Canada. Tuberculosis in Canada 2012. Ottawa (Canada): Minister of Public Works and Government Services Canada; 2015.

(2) Public Health Agency of Canada. Tuberculosis in Canada 2013-Pre-release. Ottawa, ON: Minister of Public Works and Government Services Canada; 2015.

(3) Public Health Agency of Canada. Tuberculosis Prevention and Control in Canada-A Federal Framework for Action. Ottawa, ON: Minister of Public Works and Government Services Canada; 2014. http://www.phac-aspc.gc.ca/tbpc-latb/pubs/tpc-pct/index-eng.php

(4) Menzies R, Wong T, editors. Ottawa, ON: Minister of Health; 2013. Canadian Tuberculosis Standards. 7th ed. Canadian Thoracic Society, Canadian Lung Association Public Health Agency of Canada, http://www.respiratoryguidelines.ca/tb-standards-2013 (Disponible en français : http://www.lignesdirectricesrespiratoires.ca/normes-antituberculeuse-2013).

(5) Canadian Institute for Health Information (CIHI). Canadian Coding Standards for ICD-10-CA and CCI. Ottawa, ON: ClHI; 2003. https://secure.cihi.ca/estore/productSeries.htm?locale=fr\&pc=PCC189

(6) World Health Organization (WHO). Manual of the 34th international classification of diseases, injuries, and causes of death, based on the recommendations of the ninth revision conference, 1975, and adopted by the twenty-ninth World Health Assembly. Geneva: WHO; 1977.

(7) World Health Organization. Global tuberculosis report 2014. www.who.int/tb/publications/global_report/en/. (Disponible en français: www.who.int/tb/publications/global_report/fr/). 